\section{Commentary: Do old rules apply to new valves?}

\author{
Nels D. Carroll, MD, and Dawn S. Hui, MD
}

The growing body of evidence regarding the efficacy and safety of transcatheter devices ${ }^{1,2}$ is at the forefront of a revolution. Meanwhile, surgically implanted valves continue to undergo an evolution. Competition has stimulated improvements in materials and designs at an unprecedented rate. US Food and Drug Administration approval of lower anticoagulation targets after the Prospective Randomized On-X Anticoagulation Clinical Trial for the On-X mechanical valve (CryoLife, Inc, Kennesaw, Ga) represents a significant step forward. ${ }^{3}$ Meanwhile, surgically implanted bioprosthetic valves continue to represent a large share of contemporary aortic valve interventions. Within this segment of the market, the Avalus bioprosthetic valve (Medtronic, Minneapolis, Minn) is a novel low-profile, stented bovine pericardial valve, the safety and efficacy of which has been investigated in the PERIGON trial (PERIcardial SurGical AOrtic Valve ReplacemeNt Pivotal Trial). Early results, previously reported, ${ }^{4,5}$ included favorable hemodynamics and acceptable rates of death, paravalvular leak, thromboembolism, and endocarditis. The rate of bleeding events, however, has been higher than expected.

On publication of these results, various explanations for these bleeding events were proposed. These included concomitant antithrombotic therapy for comorbidities and the suggestion that early bleeding is more likely and therefore skews the linearized projections. ${ }^{4,5}$ In this edition of the Journal, Klautz and colleagues ${ }^{6}$ address the topic of elevated bleeding risk associated with the Avalus valve more directly and report on midterm data regarding valvular deterioration. Included are 1115 patients, now with 3-year follow-up. The reported incidence of all bleeding events is $8.7 \%$, and that of major bleeding events is $5.2 \%$, as calculated from 99 cumulative events. These rates are higher than

From the Department of Cardiothoracic Surgery, University of Texas Health Science Center at San Antonio, San Antonio, Tex.

Disclosures: Authors have nothing to disclose with regard to commercial support.

Received for publication Oct 17, 2019; revisions received Oct 17, 2019; accepted for publication Oct 17, 2019; available ahead of print Oct 31, 2019.

Address for reprints: Dawn S. Hui, MD, 7703 Floyd Curl Dr, Suite 211L, San Antonio, TX 78229 (E-mail: huid@uthscsa.edu).

J Thorac Cardiovasc Surg 2021;161:76-7

$0022-5223 / \$ 36.00$

Copyright $($ C 2019 by The American Association for Thoracic Surgery

https://doi.org/10.1016/j.jtcvs.2019.10.089

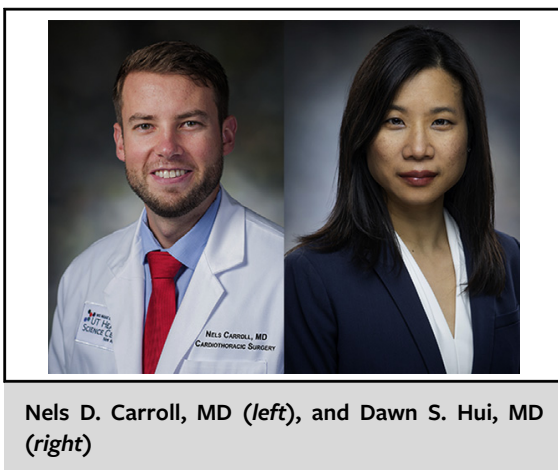

CENTRAL MESSAGE

Regulatory criteria for valve per-

formance may be affected by

therapies for unrelated comor-

bidities. The effect on clinical

practice in a changing population

is a question for the surgical

community.

the objective performance criteria (OPC) established by the International Standards Organization. Most patients who had bleeding events while on antithrombotic therapy were receiving that therapy for an indication independent of the valve.

In examining thrombotic and bleeding events as an outcome, there are 3 separate issues: the prosthesis itself, valve-related antithrombotic practices, and valveindependent comorbidities that require antithrombotic therapy. In this context, the study has notable limitations. First, although PERIGON was a clinical trial, it did not specify a protocol for postoperative antithrombotic therapy. Second, bleeding event analysis included only those patients receiving antiplatelet or anticoagulant medications. By using the OPC as the standard, Klautz and colleagues ${ }^{6}$ frame the question as such: Are OPCs outdated, or should antithrombotic and anticoagulant practices be changed? We find that the exclusion criteria and lack of a protocol restrict the study from being able to answer these questions.

From the regulatory standpoint, however, an interesting point is raised. As Klautz and colleagues ${ }^{6}$ note, "the OPC functions as an alarm in case new prostheses result in inferior outcomes compared to current prostheses." Consequently, the OPC may mistakenly shade the perception of valve safety by incorrectly ascribing bleeding hazards to valve-related practices. Whether regulatory thresholds 
affect clinical practice remains to be seen, because this issue does not seem unique to the Avalus valve.

Longer term data will provide more clarity regarding bleeding events after implantation of this valve and its competitors. The prevalence of antithrombotic therapy for comorbidities, however, simply reflects a real and diverse contemporary patient population. The rules will be slow to change, but this discussion may aptly guide our interpretation of these results.

\section{References}

1. Mack MJ, Leon MB, Thourani VH, Makkar R, Kodali SK, Russo M, et al. Transcatheter aortic-valve replacement with a balloon-expandable valve in low-risk patients. N Engl J Med. 2019;380:1695-705.
2. Popma JJ, Deeb GM, Yakubov SJ, Mumtaz M, Gada H, O’Hair D, et al; Evolut Low Risk Trial Investigators. Transcatheter aortic-valve replacement with a self-expanding valve in low-risk patients. N Engl J Med. 2019;380:1706-15.

3. Puskas J, Gerdisch M, Nichols D, Quinn R, Anderson C, Rhenman B, et al Reduced anticoagulation after mechanical aortic valve replacement: interim results from the prospective randomized on- $\mathrm{X}$ valve anticoagulation clinical trial randomized food and drug administration investigational device exemption trial. J Thorac Cardiovasc Surg. 2014;147:1202-10; discussion 1210-11.

4. Klautz RJM, Kappetein AP, Lange R, Dagenais F, Labrousse L, Bapat V, et al; PERI GON Investigators. Safety, effectiveness and haemodynamic performance of a new stented aortic valve bioprosthesis. Eur J Cardiothorac Surg. 2017;52:425-31.

5. Sabik JF III, Rao V, Lange R, Kappetein AP, Dagenais F, Labrousse L, et al; PERIGON Investigators. One-year outcomes associated with a novel stented bovine pericardial aortic bioprosthesis. J Thorac Cardiovasc Surg. 2018;156: 1368-77.e5.

6. Klautz RJM, Vriesendorp MD, Dagenais F, Labrousse L, Bapat V, Moront MG, et al. Antithrombotic therapy and bleeding events after aortic valve replacement with a novel bioprosthesis. J Thorac Cardiovasc Surg. 2021;66-75.e4. 